Nursing

\section{Working together, sharing the burden}

\section{J Perrin}

\section{The developing role of emergency department nurses}

D emands made on the health service have increased over recen years. This is most acutely felt in emergency care. Emergency dpartment nurses have had a huge impact on care delivery, some would argue that this is attributable to the motivation and concern for patient care. Others might say that it is in response to the reduction in junior doctors hours. I would suggest that it is a combination of both, in conjunction with the political driving force of the government targets. The health service is developing at such a pace it is sometimes difficult to keep up. This was illustrated at a conference recently by a piece of on screen animation whereby an airplane, which represented the NHS, was flying along while still being built!

The desire to provide a better service for patients has brought opportunities for the development of nurse led services such as minor injury units, walk in centres, and NHS Direct, integrating in many cases with their local emergency department. Further developments have been accelerated by the creation of the Emergency Services Collaborative. Expertise from staff involved in healthcare delivery is harnessed and turned into benefits for the patient. Using the "hearts and minds" approach, (with often little or no financial input), can sometimes make major improvements to the patient experience for example "See and Treat".

The EM welcomes papers on nursing issues and we hope that as they take on more expanded roles nurses will find more and more of the content of the EN relevant to their practice. We encourage suggestions on how we can make the journal more relevant. Emergency care is now a team effort and we hope that the EMW can encourage joint learning and scientific inquiry. Jim Wardrope, Editor.
However, despite the introduction of many new services and innovations, there is a lack of evidence of the impact of these services on the system as a whole because of inadequate plans for evaluation being build into projects. It is rigorous thorough evaluation that will enable patients, politicians, and the rest of the health community to truly measure the impact nurses are having on healthcare delivery.

In this issue we have three excellent papers that review the effectiveness of nurses and their new roles.

The article by Marr et al maps the range and scope of emergency nurse practitioner (ENP) services in the Northern and Yorkshire region. ${ }^{2}$ This research highlights the lack of standardised training that ENP's undertake. For any new service it is essential that healthcare professionals are adequately prepared, otherwise the patient may receive substandard care and the carer exposes themselves to litigation. The recently launched Faculty of Emergency Nursing may go some way to address this issue. The study also highlights the difference in scope of practice and empowerment. The study covered a large area of the country and provides us with a good overview, this is a study that I feel should be undertaken nationally to give a complete picture of service provision. It would then be possible to work towards providing equitable care for patients attending with minor injuries throughout the UK.

The issue of ongoing competence also needs to be addressed, as at present there are no national requirements for continuing professional development.

In the field of cardiac care, many areas have undertaken to set up fast track services to deliver the target of $75 \%$ of patients receiving thrombolysis within 30 minutes of arrival to hospital. The study by Heath et al investigates whether "fast tracking" the patient to coronary care unit for thrombolysis is preferable to nurse initiated thrombolysis in the A\&E department. ${ }^{3}$ It concludes that the prescription and administration of thrombolysis by experienced cardiology nurses in A\&E provides a safe and timely strategy for door to needle times with significant benefits.

The third study by Frampton et al considers how safe and effective nurses are at administering high concentration nitrous oxide relative analgesia in children undergoing minor procedures. ${ }^{4}$

These studies highlight the effectiveness of nurses delivering high quality patient care improving the patient experience.

The development of new services is often restricted by legalities, for example nurses administering opioids. New innovations may need to be held in check until the appropriate guidelines or legal hurdles have been overcome, but at times this process seems very bureaucratic. This has a place in the protection of the patients, but is often frustrating for those attempting to deliver high quality care and make progress against the "targets".

However, nurses only make up one portion of the team caring for the patient. It is sometimes easy to focus on one particular group of health carers and for them to become disengaged with the rest of the team. We need to focus on a multiprofessional approach, value and respect each other's contributions.

Emerg Med J 2003;20:398

Author's affiliations

J Perrin, Northern General Hospital, Herries Road, Sheffield S5 7AU, UK:

julie.perrin@sth.nhs.uk

\section{REFERENCES}

1 Castille K, Cooke M. Controversies in emergency care. One size does not fit all. Emerg Med J 2003;20:120-1

2 Marr S, Steele K, Swallow V, et al. Mapping the range and scope of emergency nurse practitioner services in the Northern and Yorkshire Region: a telephone survey. Emerg Med J 2003;20:414-17

3 Heath SM, Bain RJl, Andrews A, et al. Nurse initiated thrombolysis in the accident and emergency department: safe, accurate, and faster than fast track. Emerg Med J 2003;20:418-20.

4 Frampton A, Browne GJ, Lam LT, et al. Nurse administered relative analgesia using high concentration nitrous oxide to facilitate minor procedures in children in an emergency department. Emerg Med J 2003;20:410-13. 


\section{Health care policy makers, we have a problem}

\section{J Wardrope, P Driscoll}

\section{The international crisis in emergency care}

T he papers by Trzeciak $^{1}$ and Fatovich, ${ }^{2}$ along with the commentary by Schull, ${ }^{3}$ highlight the international dimension to the problem of crowed emergency departments (ED). To deal with this problem the United Kingdom National Health Service has published ambitious plans that state that all patients should be seen, assessed, treated, and either admitted or discharged within four hours. ${ }^{4}$ As part of this initiative, department times in the EDs in England were assessed during the last week in March. The published results indicated that almost all EDs met the interim "target" of having at least $90 \%$ of patients being discharged or admitted within four hours of arrival.

The question that many are asking is what does this target mean from the patient's perspective? Few would disagree with using department times as a surrogate measure of efficiency and progress. All patients seem to take high quality care for granted and waiting time is the most important factor in most patient satisfaction surveys. Unfortunately, for many hospitals, the target itself has become the goal. Consequently extra resources and procedures were implemented only for the duration of the assessment period of one week. Some hospitals even cancelled elective surgery. ${ }^{5}$ This aim for a short-term solution rather than a whole system approach has generated much debate and criticism.

The commentary by Schull shows that it is possible to meet emergency care demands in the short-term by focusing the considerable capacity of health care systems on handling emergency admissions. The emergency rooms in Toronto were very quiet during the recent outbreak of severe acute respiratory syndrome (SARS). This was mainly due to fewer patients coming to the ED and more hospital beds being available for emergency admissions because of the cancellation of elective surgery.

ED overcrowding is a symptom that an emergency health care system cannot cope with its workload. Each component of the system, including the ED, needs to review its own practice and maximise effectiveness, but it seems that it is the availability of hospital beds that is one of the main determinants of delays for all ED patients (exit block). ${ }^{6}$ As a department becomes crowded with patients awaiting admission its efficiency plummets along an exponential curve. There is no space to see the patients and both nursing and medical staff become increasingly tied up in dealing with their ongoing care. Meanwhile the queue of new patients grows, patients become frustrated, and staff feel overwhelmed.

The fact that the same picture is emerging from many economically advanced societies, indicates that the problem has its roots in major forces within society. These include shifts in social structures, demographics, reduced capacity in the hospital sector, and in the changes of our emergency care systems.

The increased social mobility of western society has led to a weakening of the normal support structures of the extended family. Vulnerable groups, especially the elderly, come to rely on friends, neighbours or professional help. Such carers provide a huge amount of support in the community but may be less willing than extended family to deal with the added burden or risks of minor and moderate illness. Often the easiest solution seems to be a call to the emergency medical system (for example a 999 or 911 call), an ambulance arrives to provide immediate relief and the patient is taken to hospital.
During the late 1980s and 1990s there was a great drive to reduce hospital costs. Centres were closed, bed numbers were reduced, and staffing levels were minimised in the quest for efficiency. With hospitals now running at over $95 \%$ bed occupancy there is no slack to cope with emergency demand. Compounding this problem is the fact that primary care doctors are becoming less involved in out of hours, weekend, and holiday cover.

Any solutions must try to address these major social and health system changes. Simple cosmetic surgery will not succeed. Such surgery can make things look a bit better temporarily but is doomed to fail in the longer term.

We need more capacity for health care, not necessarily in hospitals but increased support and care for those living at the margins of safety in the community. Intermediate care is seen as a solution, and probably is, but we have seen little progress in making this effective.

We need to stem the demands on emergency care. There are no votes for politicians in trying to set the limits on the expectations of the population for health care. Perhaps the medical profession is also guilty of fuelling patients' ideas that medicine has the answer to every problem and can deal with every risk. Some very basic honesty is required by all or we will be discussing the problem of ED overcrowding for ever.

Emerg Med J 2003;20:399

\section{Authors' affiliations}

J Wardrope, P Driscoll, Joint Editors

Correspondence to: Mr J Wardrope; Jim.Wardrope@sth.nhs.uk

\section{REFERENCES}

1 Trzeciak S, Rivers EP. Emergency department overcrowding in the United States: an emerging threat to patient safety and public health. Emerg Med J 2003;20:402-5.

2 Fatovich DM, Hirsch RL. Entry overload, emergency department overcrowding, and ambulance bypass. Emerg Med J 2003; 20:406-9.

3 Schull MJ. Sex, SARS, and the Holy Grail. Emerg Med J 2003;20:400-1

4 Department of Health. Reforming emergency care. www.doh.gov.uk/ emergencycare/reformingemergencycare

5 Mayor S. Hospitals take short term measures to meet targets. BM 2003:326:1054.

6 Miro O, Sanchez M, Espinosa G, et al. Analysis of patient flow in the emergency department and the effect of extensive reorganisation. Emerg Med J 2003; 20: $143-8$ 